\section{A Simple Procedure for Enhancing PCR Specificity}

\section{Florian Weighardt, Giuseppe Biamonti, and Silvano Riva}

Istituto di Genetica Biochimica ed Evoluzionistica del C.N.R., I-27100

Pavia, Italy
The PCR technique is one of the most powerful tools in modern molecular genetics. It enhances greatly the potentiality of recombinant DNA technology. This technique, however, is often plagued by artifacts mostly attributable to the spurious annealing of primers to sequences only partially identical to the true recognition sites. This problem is often encountered when total genomes are used as templates given the high genetic complexity and the extremely low target-to-total DNA ratio. Moreover, if the spurious annealing of primers occurs in between the two recognition sites, smaller products, which are amplified more efficiently, become predominant over the desired product.

Several ways to overcome this difficulty have been devised. ${ }^{(1)}$ One possibility is to employ a different set of primers until a satisfactory result is obtained. However, this solution is laborious and is not practicable when the amplified DNA must end at fixed borders (e.g., for subsequent cloning in an expression vector). Another way to increase PCR specificity is to increase as much as possible the annealing temperature and/or add formamide to the reaction mixture. ${ }^{(2)}$ Usually, this procedure improves the specificity of the reaction but is not effective when the two primers have different annealing temperatures. This occurs because annealing conditions that are stringent for the primer with lower melting temperature may be too relaxed for the other primer. It should be outlined that while primers should ideally have the same melting temperature values, experimental restraints (e.g., primer-dimer formation) and/or practical considerations can sometimes lead to the use of primers with different melting temperature.

The PCR method described in this paper seems to have overcome most of the problems mentioned above. It also yielded the expected products, virtually uncontaminated, in cases where the standard PCR procedures failed. The key features of our method are the following: (1) tailed primers, i.e. oligonucleotides containing 15-20 nucleotides complementary to the recognition sites on the template DNA tailed at their 5'ends with an unrelated sequence of 10-15 nucleotides (the entire primer should be at $T_{\mathrm{m}}>72^{\circ} \mathrm{C}$ ); and (2) a twostep amplification reaction, that is, sep- arate primer extensions with each primer at its highest annealing temperature followed by PCR cycles in which annealing and extension temperatures coincide $\left(72^{\circ} \mathrm{C}\right)$. In the first step of the reaction, only a few molecules containing the tailed primers at their 5 '-end are synthesized. The entire primers in turn become new and unique high-stringency recognition sites in the following PCR cycles. Because for obvious considerations, the probability of recognition sites for the entire primers in the original DNA template is close to zero, the latter is excluded from the subsequent PCR cycles (therefore minimizing the possibility of aspecific priming) and replaced by the molecules produced by the very first primer extensions.

The proposed methodology is depicted schematically in Figure 1 where, for the sake of generality, the most unfavorable situation is considered, i.e., when the annealing temperatures of the two oligonucleotides to their target sequences are significantly different. In the case of primers with the same melting temperature, an obvious simplification of the procedure is possible.

The first step (Fig. 1, step 1) entails a series of primer extensions (about five) of the oligonucleotide (oligo 1 ) with the lower annealing temperature $\left(\mathrm{T}_{1}\right)$, and produces molecules with oligo 1 at their 5 '-end.

In the next step (Fig. 1, step 2) the second oligonucleotide (oligo 2) is added to the reaction and five PCR cycles are performed at the annealing temperature $\left(T_{2}\right)$ of oligo $2\left(T_{2}>T_{1}\right)$. The first of these PCR cycles is actually a primer extension reaction of oligo 2 that produces the two types of molecules indicated by A and B. From the second cycle on, a true PCR occurs where oligo 1 anneals over its entire length to the newly synthesized template of type $B$, producing the type of molecules indicated by type C.

The last step (Fig. 1, step 3) is a highstringency two-step PCR ( 35 cycles) at a temperature $\left(72^{\circ} \mathrm{C}\right)$ that imposes annealing of the entire oligonucleotides and is optimal for the Taq polymerase extension. Such reactions produce type $\mathrm{C}$ molecules only.

Our method minimizes spurious hybridizations both by annealing short sequences at their highest allowed temperature and by minimizing the number of 


\section{Technical TipsIIIIII}

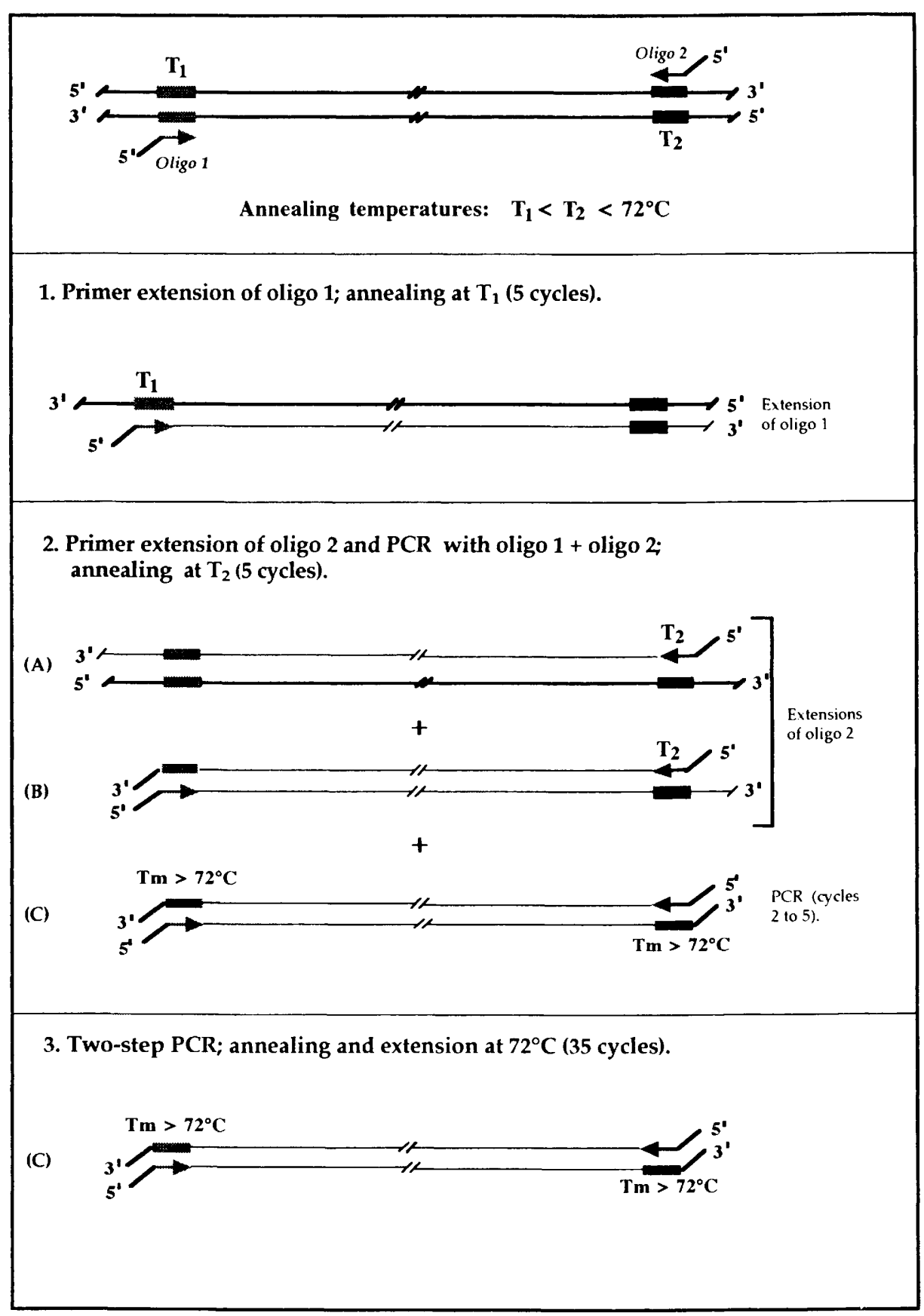

FIGURE 1 Schematic outline of the modified PCR procedure. Stippled and solid boxes represent primer recognition sites on the template. Bent arrows represent the oligonucleotide primers, with the black tilted bar indicating the $5^{\prime}$-end tail. $T_{1}$ and $T_{2}$ represent the optimal annealing temperatures of oligonucleotides 1 and 2 , respectively, to their recognition sites on the template. The reaction steps and type $A, B$, and $C$ molecules are discussed in the text.

such annealings. After the first primer extensions, new templates are produced containing recognition sites longer than the original ones and with no correspondence in the initial template. Hence, in the following cycles at the annealing temperature of $72^{\circ} \mathrm{C}$, only these new templates are amplified while the bulk of initial DNA is excluded from the reaction.

The proposed method was tested in two cases in which standard PCR procedures gave unsatisfactory results with a prevalence of undesired spurious prod- ucts. The first case concerned the amplification of a region of the human genome encompassing one intron of the human heterogenous nuclear ribonucleoprotein (hnRNP) A1 gene ${ }^{(3)}$ using primers with recognition sequences in the two flanking exons. As shown in Figure $2 \mathrm{~A}$, the standard PCR (with both sets of primers, normal and tailed ones) yielded very little of the expected 330-nucleotide band contaminated by a great number of spurious fragments of different sizes. This result is probably attributable to the fact that the hnRNP Al gene belongs to a multigene family with numerous pseudogenes scattered in the genome, ${ }^{(3)}$ many of which can be recognized by the primers and yield artifactual amplification products. Moreover, sequence analysis revealed that one of the two primers has possible additional recognition sites in downstream exons (data not shown). The addition of formamide ${ }^{(2)}$ improved the specificity of the reaction only slightly (see Fig. 2A). On the contrary, when the same region was amplified by our procedure as described previously, a significantly better result was obtained (see Fig. 2B). The expected band was clearly prominent and virtually free of contaminating products.

The other case investigated was the amplification of an internal region of human lamin B2 cDNA encoding the nuclear localization signal of the protein. ${ }^{(4)}$ For this experiment a truncated lamin B2 cDNA cloned in pUC19 plasmid was used as template with two primers (see the legend to Fig. 3) flanking the region of interest. As shown in Figure 3 (left), with the standard PCR protocol several smaller bands were produced by the reaction, in addition to the expected 155 nucleotide band. Also in this case, the amplification of this region with our modified procedure eliminated the spurious products and yielded only the desired band (see Fig. 3, right).

Interestingly, we found that a slight modification of the method allowed the addition of both oligonucleotides at the beginning of the reaction. In this case, only one primer extension is performed at the annealing temperature $\left(T_{1}\right)$ for oligo 1 , followed by five PCR cycles at the annealing temperature $\left(T_{2}\right)$ for oligo 2 and, finally, by 35 cycles of high-stringency PCR (annealing temperature, $72^{\circ} \mathrm{C}$ ). The first primer extension reaction (at $T_{1}$ ) generates two types of extended molecules; one from oligo 1 an- 


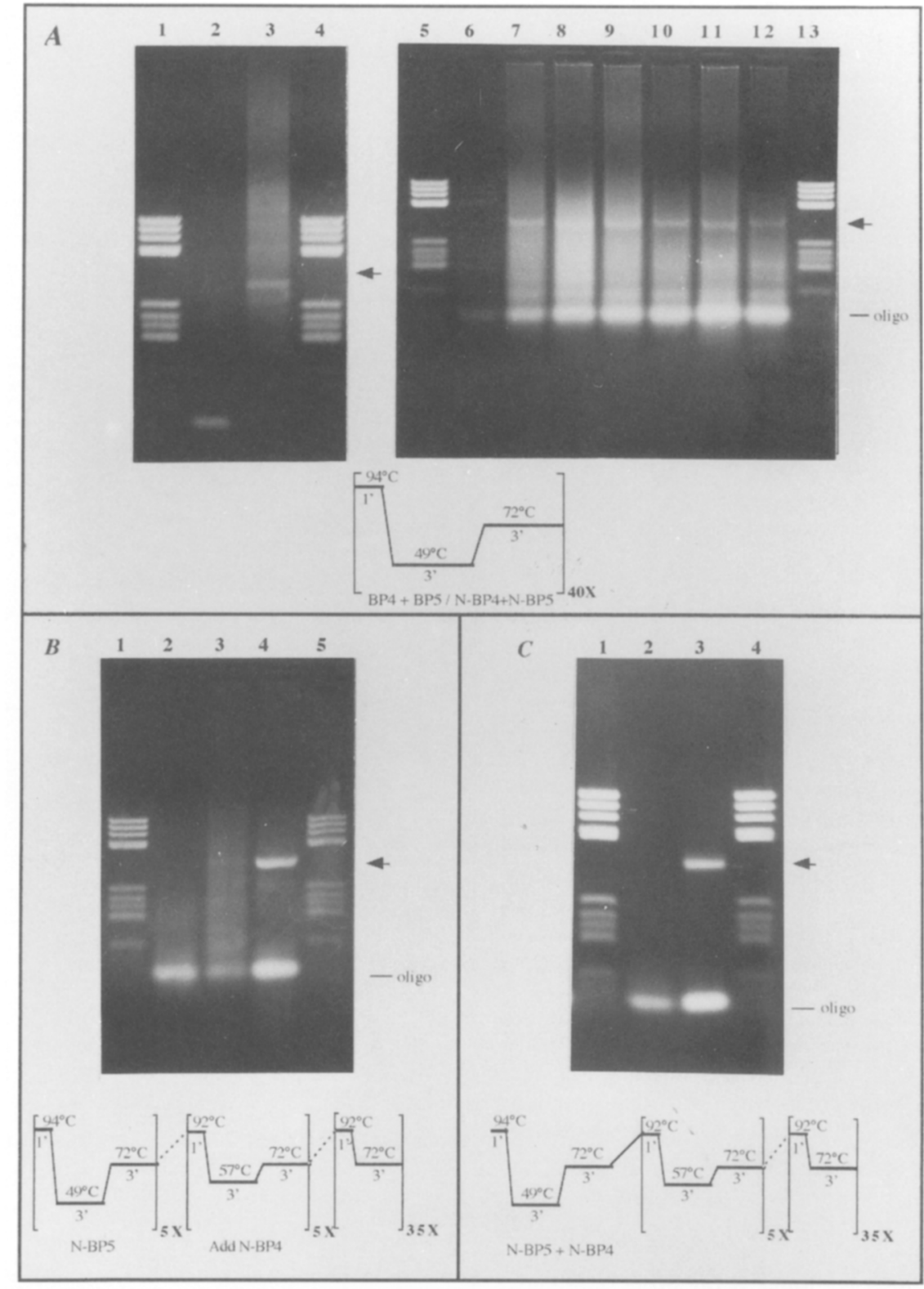

FICURE 2 PCR amplification of a region of the human genome. A pair of 19-nucleotide-long oligonucleotides, BP4 (5'-ACGCTCACGGACTGTGTGG-3') and BP5 (5'-GTTTGGATCTCTCATTACC-3'), were synthesized complementary to sequences in exons 5 and 6 and flanking the fifth intron of the hnRNP A1 gene. The distance between the two target sites on the template is 330 nucleotides. The modified (tailed) oligonucleotides N-BP4 and N-BP5 (36 nucleotides) were obtained by adding the sequence (unrelated to the template) 5'-GGCATAGCTGAATGCAT-3' to the $5^{\prime}$-end of both BP4 and BP5. The underlined sequence is the recognition site for the restriction endonuclease Nsil. The calculated melting temperatures for BP4 and BP5 are $62^{\circ} \mathrm{C}$ and $52^{\circ} \mathrm{C}$, respectively, while those of the tailed oligonucleotides N-BP4 and N-BP5 are $>72^{\circ} \mathrm{C}$. PCR amplifications were performed using a Perkin-Elmer AmpliTaq kit in the presence of $1.2 \mu \mathrm{g}$ of HL-60 cellular DNA and $1.5 \mu \mathrm{g}$ of each oligonucleotide. The $100-\mu \mathrm{l}$ reactions were run in a Perkin-Elmer Thermal Cycler according to the schemes shown. (A) Standard PCR with normal primers (BP4 and BP5) or tailed primers (N-BP4 and N-BP5) performed as in the scheme below. (Lanes 1,4,5,13) Molecular weight markers (pBR322/HaeIII); (lanes 2,6) no DNA; (lane 3) standard PCR with primers BP4 and BP5; (lane 7) standard PCR with primers N-BP4 and N-BP5; (lane 8-12) standard PCR with primers N-BP4 and N-BP5 in presence of $1-5 \%$ formamide. The arrow indicates the position of the expected band. The positions of unextended oligonucleotides are shown also. $(B)$ Modified PCR (see Fig. 1) with tailed primers N-BP4 and N-BP5. (Lane 1,5) Molecular weight markers (pBR322/HaeIII); (lane 2) no DNA; (lane 3) standard PCR as in A; (lane 4) modified PCR as in the scheme below (see Fig. 1). (C) Modified PCR with both tailed primers present from the beginning of the reaction (see text). (Lanes 1,4) Molecular weight markers (pBR322/HaeIII); (lane 2) no DNA; (lane 3) modified PCR as in the scheme below. nealed at high stringency (Fig. 1, type B), and the other from oligo 2 (type A) annealed at low stringency. While the first type is enriched for the desired molecules, the second can be contaminated by molecules derived from spurious primings of oligo 2 . Analogous to what is described above, in the next five cycles at $T_{2}$ only type $B$ molecules can be amplified exponentially. On the contrary, type A molecules increase only linearly because $T_{2}$ is not permissive for the annealing of oligo 1 . The final 35 cycles at high-stringency PCR (annealing temperature, $72^{\circ} \mathrm{C}$ ) will select further for amplification of the products of the initial primer extension with oligo 1 and yield only type $\mathrm{C}$ molecules (see Fig. 1).

When this procedure was tested in the amplification of the human hnRNP A1 protein intron, the same result as that observed with the two oligonucleotides added stepwise was observed (Fig. 2, cf. C and $\mathrm{B}$ ).

In conclusion, the PCR procedure described in this paper drastically improved the specificity of the reaction under conditions where standard PCR procedures yielded predominantly spurious products. In principle, this method can solve many of the problems caused by spurious hybridization of primers and may be helpful in the amplification of sequences belonging to multigene families. From an applicative point of view, our procedure is very simple and requires no preliminary work when, as is often the case, primers are already tailed at their 5 '-end with restriction enzyme sites for subsequent cloning. ${ }^{(1,5)}$

To this regard it should be mentioned that cloning of PCR fragments is often plagued by the presence of spurious product. Methods have been devised to select for the desired product such as those ${ }^{(6)}$ based on the use of an additional set of primers in a second PCR. Our method can instead attain the same result in one step and with a single set of primers.

\section{ACKNOWLEDGMENTS}

This work was funded partially by the Progetto Finalizzato Ingegneria Genetica C.N.R., Rome. F.W. was supported by a fellowship of the Fondazione Adriano Buzzati-Traverso. 


\section{Technical TipsIIIIIIII}

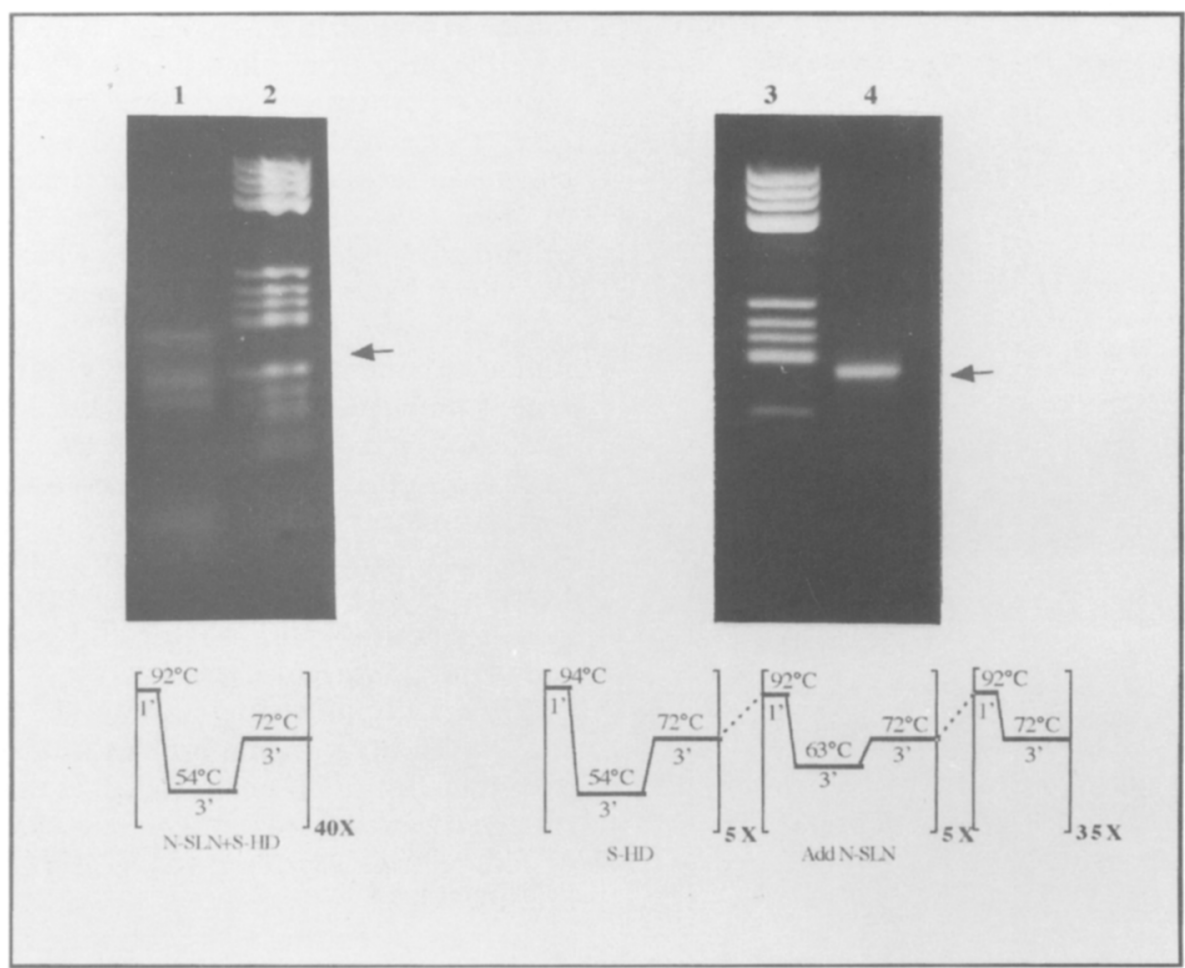

FICURE 3 PCR amplification of a region of the cDNA for the human lamin B2 protein cloned in a pUC19 plasmid. A pair of 36-nucleotide-long tailed oligonucleotides, N-SLN and S-HD, were synthesized complementary to the sequences of the region of human lamin B2 cDNA encoding the nuclear localization signal. The distance on the template between the two oligonucleotides is 129 nucleotides. The sequence of $\mathrm{N}$-SLN is $5^{\prime}$-GGCAGGCATTCA-ATGCAT-AGCCCTTGGCAG CGGCCC-3'. The underlined sequence is the recognition sequence for the restriction endonuclease Nsil. The calculated melting temperature for annealing of N-SLN to the template is $64^{\circ} \mathrm{C}$. The sequence of S-HD is 5'-GGCAGGCATTCA-GTCGAC-CACAAACTTGCCCTCCAG-3'. The underlined sequence is the recognition sequence for the restriction endonuclease Sall. The calculated melting temperature for the annealing of S-HD to the template is $56^{\circ} \mathrm{C}$. PCR amplification mixtures $(100 \mu \mathrm{l})$ contained $10 \mathrm{ng}$ of plasmid DNA and $1.5 \mu \mathrm{g}$ of each oligonucleotide. Reactions were run according to the schemes below. (Lane 1) Standard PCR; (lanes 2,3) molecular weight markers (pBR322/HaeIII); (lane 4) modified PCR.

\section{REFERENCES}

1. Innis, M.A., D.H. Gelfand, J.J. Sninsky, and T.J. White. 1990. PCR protocols: $A$ guide to methods and applications. Academic Press, San Diego, CA.

2. Sarkar, G., S. Kapelner, and S. S. Sommer. 1990. Formamide can dramatically improve the specificity of PCR. Nucleic Acids Res. 18: 7465.

3. Biamonti, G., M. Buvoli, M.T. Bassi, C. Morandi, F. Cobianchi, and S. Riva. 1989. Isolation of an active gene encoding human hnRNP protein A1. Evidence for alternative splicing. J. Mol. Biol. 207: 491503.

4. Biamonti, G., M. Giacca, G. Perini, G. Contreas, L. Zentilin, F. Weighardt, M. Guerra, G. Della Valle, S. Saccone, S. Riva, and A. Falaschi. 1992. The gene for a novel human lamin maps at a highly transcribed locus of chromosome 19 which replicates at the onset of S-phase. Mol. Cell. Biol. 12: 3499-3506.

5. Arnheim, N. and H. Erlich. 1992. Polymerase chain reaction strategy. Annu. Rev. Biochem. 61: 131-156.

6. Lo, Y.-M.D., P. Patel, J.S. Wainscoat, M. Sampietro, D.G. Gillmer, and K.A. Fleming. 1989. Prenatal sex determination by DNA amplification from maternal peripheral blood. Lancet ii: 1363-1365.

Received April 22, 1993; accepted in revised form June 21, 1993. 


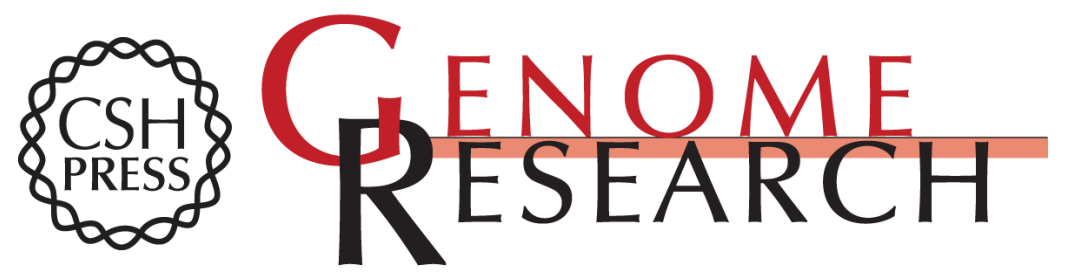

\section{A simple procedure for enhancing PCR specificity.}

F Weighardt, G Biamonti and S Riva

Genome Res. 1993 3: 77-80

References This article cites 4 articles, 1 of which can be accessed free at:

http://genome.cshlp.org/content/3/1/77.full.html\#ref-list-1

\section{License}

Email Alerting Receive free email alerts when new articles cite this article - sign up in the box at the Service top right corner of the article or click here.

\section{Affordable, Accurate Sequencing.}

To subscribe to Genome Research go to: https://genome.cshlp.org/subscriptions 